\title{
Polarimetric Edge Detector based on the complex Wishart distribution
}

\author{
Henning Skriver ${ }^{1}$, Jesper Schou ${ }^{1}$, Allan Aasbjerg Nielsen ${ }^{2}$, and Knut Conradsen ${ }^{2}$ \\ ${ }^{1}$ Section for Electromagnetic Systems, Ørsted•DTU Department \\ Technical University of Denmark, Building 348, DK-2800 Lyngby, Denmark \\ Phone: +45 458814 44, Fax: +45 459316 34, E-mail: hs@oersted.dtu.dk ${ }^{1}$ \\ ${ }^{2}$ Informatics, and Mathematical Modelling Department \\ Technical University of Denmark, Building 321, DK-2800 Lyngby, Denmark
}

\begin{abstract}
A new edge detector for polarimetric SAR data has been developed. The edge detector is based on a newly developed test statistic for equality of two complex covariance matrices following the complex Wishart distribution and an associated asymptotic probability for the test statistic. The new polarimetric edge detector provides a constant false alarm rate and it utilizes the full polarimetric information. The edge detector has been applied to polarimetric SAR data from the Danish dual-frequency, airborne polarimetric SAR, EMISAR. The results show clearly an improved edge detection performance for the full polarimetric detector compared to single channel approaches.
\end{abstract}

\section{INTRODUCTION}

Edge detection is an important image processing operation that is for instance used for structure detection, as part of segmentation algorithms and of adaptive filters. Edge detection is a low-level operation, i.e. it is very important that the edge detector is adapted to the image statistics. In the middle of the eighties the ratio edge detector was developed for single channel SAR images [1][2]. Using the Gamma distribution for the homogeneous areas in single channels SAR images, the ratio detector provides a constant false alarm rate (CFAR), where the false alarm rate is the probability of detecting a non-existing edge in a homogeneous area. This detector has been widely used with success by the SAR community in a large number of cases.

In this paper, a new edge detector is presented that provides a constant false alarm rate for polarimetric SAR data. For a homogeneous surface, polarimetric SAR data are completely described by the complex covariance matrix [3]. One approach to edge detection in polarimetric SAR images, is to perform the detection separately for each polarization, and subsequently combine the results using some kind of fusion operator [4][5]. This approach, however, does not utilize the full polarimetric information, as only the backscattering coefficients are used. Also, the choice of the fusion operator affects the final result. In this work, we describe a new approach to edge detection in polarimetric SAR data using a newly developed test for equality of complex covariance matrices following a complex Wishart distribution [6][7]. By estimating the covariance matrices on each side of the central pixel, we test whether these two covariance matrices are equal, and edges are detected when the hypothesis is rejected. A more thorough description of the edge detector is provided in [8].

\section{TEST STATISTIC}

This section briefly describes the complex Wishart distribution, and the likelihood ratio test for equality of two complex Wishart matrices. For a more thorough description, see [6][8].

\section{A. Complex Wishart distribution}

A fully polarimetric SAR measures the $2 \times 2$ complex scattering matrix at each resolution cell. If $\mathrm{S}_{\mathrm{ij}}$ denotes the complex scattering amplitude for receive and transmit polarization $\mathrm{i}$ and $\mathrm{j}$ ( $\mathrm{ij} \in\{\mathrm{h}, \mathrm{v}\}$ ), then reciprocity, which normally applies to natural targets, gives $\mathrm{S}_{\mathrm{hv}}=\mathrm{S}_{\mathrm{vh}}$ (in the backscattering direction using the backscattering alignment convention) [3]. Assuming reciprocity the scattering matrix is represented by the 3-component complex target vector $\mathbf{s}=$ $\left[\mathrm{S}_{\mathrm{hh}} \mathrm{S}_{\mathrm{hv}} \mathrm{S}_{\mathrm{vv}}\right]^{\mathrm{t}}$, where $\mathrm{t}$ denotes transpose. $\mathbf{s}$ follows a zero mean multivariate Gaussian distribution [9]. The sample covariance matrix $\mathbf{C}$ is formed from the target vector by

$$
\begin{aligned}
\mathbf{C} & =4 \pi\left\langle\mathbf{s s}^{{ }^{*} \mathrm{t}}\right\rangle \\
& =\left[\begin{array}{ccc}
\sigma_{\mathrm{hh}} & \sqrt{\sigma_{\mathrm{hh}} \sigma_{\mathrm{hv}}} \rho_{\mathrm{hh}, \mathrm{hv}} & \sqrt{\sigma_{\mathrm{hh}} \sigma_{\mathrm{vv}}} \rho_{\mathrm{hh}, \mathrm{vv}} \\
\sqrt{\sigma_{\mathrm{hh}} \sigma_{\mathrm{hv}}} \rho_{\mathrm{hh}, \mathrm{hv}}^{*} & \sigma_{\mathrm{hv}} & \sqrt{\sigma_{\mathrm{hv}} \sigma_{\mathrm{vv}}} \rho_{\mathrm{hv}, \mathrm{vv}} \\
\sqrt{\sigma_{\mathrm{hh}} \sigma_{\mathrm{vv}}} \rho_{\mathrm{hh}, \mathrm{vv}}^{*} & \sqrt{\sigma_{\mathrm{hv}} \sigma_{\mathrm{vv}}} \rho_{\mathrm{hv}, \mathrm{vv}}^{*} & \sigma_{\mathrm{vv}}
\end{array}\right](1)
\end{aligned}
$$

where $*$ denotes the complex conjugate, $\rho_{\mathrm{i}, \mathrm{j}}$ is the complex correlation coefficient between channels $i$ and $j$, and $\sigma$ is the backscatter coefficient.

If the $\mathrm{p} \times \mathrm{p}$ sample covariance matrix has $\mathrm{L}$ number of looks, we define the Hermitian matrix $\mathbf{Z}=\mathrm{L} \mathbf{C}$. $\mathbf{Z}$ follows a complex Wishart distribution $\mathrm{W}_{\mathrm{C}}(\mathrm{p}, \mathrm{L}, \Sigma)$ having mean covariance matrix $\Sigma$ [9], i.e.

\footnotetext{
${ }^{1}$ The work has been supported by the Technical University of Denmark and the Danish ESA Follow-on Research Board. The data acquisition and SAR processing have been supported by the Danish National Research Foundation.
} 


$$
\mathrm{p}(\mathbf{Z} \mid \Sigma)=\frac{|\mathbf{Z}|^{\mathrm{L}-\mathrm{p}} \mathrm{e}^{-\operatorname{tr}\left(\Sigma^{-1} \mathbf{Z}\right)}}{\pi^{\frac{1}{2} \mathrm{p}(\mathrm{p}-1)} \sum_{\mathrm{j}=1}^{\mathrm{p}} \Gamma(\mathrm{L}-\mathrm{J}+1) \mid \Sigma^{\mathrm{L}}}
$$

where || and $\operatorname{tr}()$ denote the determinant and the trace, respectively, and $\Gamma()$ is the Gamma function.

\section{$B$ Test for equality of complex covariance matrix}

In [6][7] a test statistic in the complex Wishart distribution have been presented, where the likelihood ratio test us used for testing the equality of two complex Wishart distributed matrices. If $\mathbf{Z}_{\mathrm{x}}$ and $\mathbf{Z}_{\mathrm{y}}$ follow the complex Wishart distributions $\mathrm{W}_{\mathrm{C}}\left(\mathrm{p}, \mathrm{L}_{\mathrm{x}}, \Sigma_{\mathrm{x}}\right)$ and $\mathrm{W}_{\mathrm{C}}\left(\mathrm{p}, \mathrm{L}_{\mathrm{y}}, \Sigma_{\mathrm{y}}\right)$, respectively, then the null hypothesis $\mathrm{H}_{0}: \Sigma_{\mathrm{x}}=\Sigma_{\mathrm{y}}$ against the alternative hypothesis $\mathrm{H}_{1}: \Sigma_{\mathrm{x}} \neq \Sigma_{\mathrm{y}}$ states, that the two matrices $\mathbf{Z}_{\mathrm{x}}$ and $\mathbf{Z}_{\mathrm{y}}$ are equal [6][7]. The Wishart likelihood test statistic becomes

$$
\mathrm{Q}=\frac{\left(\mathrm{L}_{\mathrm{x}}+\mathrm{L}_{\mathrm{y}}\right)^{\mathrm{p}\left(\mathrm{L}_{\mathrm{x}}+\mathrm{L}_{\mathrm{y}}\right)}}{\mathrm{L}_{\mathrm{x}}^{\mathrm{pL}_{\mathrm{x}}} \mathrm{L}_{\mathrm{y}}^{\mathrm{p \textrm {L } _ { \mathrm { y } }}}} \frac{\left|\mathbf{Z}_{\mathrm{x}}\right|^{\mathrm{L}_{\mathrm{x}}}\left|\mathbf{Z}_{\mathrm{y}}\right|^{\mathrm{L}_{\mathrm{y}}}}{\left|\mathbf{Z}_{\mathrm{x}}+\mathbf{Z}_{\mathrm{y}}\right|^{\mathrm{L}_{\mathrm{x}}+\mathrm{L}_{\mathrm{y}}}}
$$

and the asymptotic distribution of $-2 \rho \log Q$ is given as

$$
\begin{aligned}
& \mathrm{P}\{-2 \rho \log \mathrm{Q} \leq \mathrm{z}\} \cong \mathrm{P}\left\{\chi^{2}(\mathrm{f}) \leq \mathrm{z}\right\}+ \\
& \omega_{2}\left[\mathrm{P}\left\{\chi^{2}(\mathrm{f}+4) \leq \mathrm{z}\right\}-\mathrm{P}\left\{\chi^{2}(\mathrm{f}) \leq \mathrm{z}\right\}\right]
\end{aligned}
$$

with the parameters $f, \rho$ and $\omega_{2}$ defined as

$$
\begin{gathered}
f=\mathrm{p}^{2} \\
\rho=1-\frac{2 \mathrm{p}^{2}-1}{6 \mathrm{p}}\left(\frac{1}{\mathrm{~L}_{\mathrm{x}}}+\frac{1}{\mathrm{~L}_{\mathrm{y}}}+\frac{1}{\mathrm{~L}_{\mathrm{x}}+\mathrm{L}_{\mathrm{y}}}\right) \\
\omega_{2}=-\frac{\mathrm{f}}{4}\left(1-\frac{1}{\rho}\right)^{2}+\frac{\mathrm{f}(\mathrm{f}-1)}{24}\left(\frac{1}{\mathrm{~L}_{\mathrm{x}}^{2}}+\frac{1}{\mathrm{~L}_{\mathrm{y}}^{2}}+\frac{1}{\left(\mathrm{~L}_{\mathrm{x}}+\mathrm{L}_{\mathrm{y}}\right)^{2}}\right) \frac{1}{\rho^{2}}
\end{gathered}
$$

The term $-2 \rho \log Q$ belongs to the interval $[0, \infty[$, with $2 \rho \log \mathrm{Q}=0$ for $\mathbf{Z}_{\mathrm{x}}=\mathbf{Z}_{\mathrm{y}}$ and $\mathrm{L}_{\mathrm{x}}=\mathrm{L}_{\mathrm{y}}$.

The elements in the covariance matrix containing co- and cross-polarized components often contain little information, and for randomly distributed targets with azimuthal symmetry, these elements are zero for the mean covariance matrix [10], i.e. for $\Sigma$. It is important to notice, however, that if the sample covariance matrix is forced into azimuthal symmetry, i.e. if the elements containing co- and crosspolarized components in the sample covariance matrix are set to zero, then the sample covariance matrix no longer follows the complex Wishart distribution [6]. In [6] and [8] the test statistic and the asymptotic distribution are derived for the azimuthal symmetric case, as well as for the diagonal case, where all off-diagonal elements are set equal to zero.

\section{EDGE DETECTOR}

The edge detection is performed by visiting each pixel in the image in succession, and for each pixel applying a set of filters having different orientation. The filters estimate the mean covariance matrices on each side of the central pixel, and the test of equality of these two mean covariance matrices determines whether an edge is detected by the current filter or not. To ensure the greatest amount of flexibility, the edge detection filters are defined according to Fig. 1. A filter configuration, $\mathrm{K}_{\mathrm{f}}=\left\{1_{\mathrm{f}}, \omega_{\mathrm{f}}, \mathrm{d}_{\mathrm{f}}, \Delta \mathrm{v}\right\}$, is described by the length, the width, the spacing of the filter, and the angular increment $\Delta \mathrm{v}$ between two orientations. $\mathrm{N}_{\mathrm{f}}$ $=\pi / \Delta \mathrm{v}$ filters exist for each filter configuration.

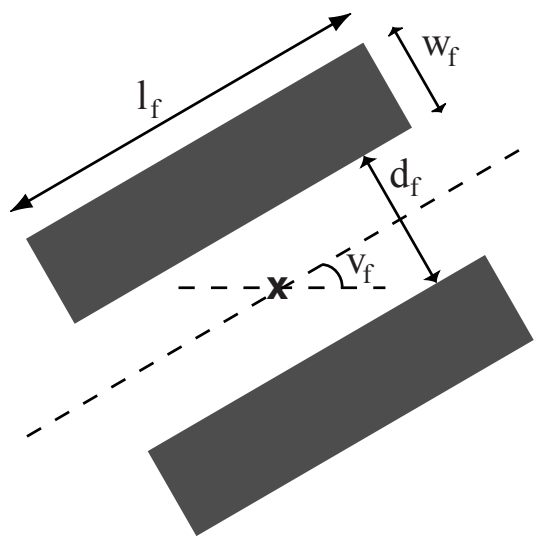

Fig. 1 Edge detector filter configuration

\section{RESULTS}

The edge detector has been tested on fully polarimetric SAR data from the Danish, airborne polarimetric SAR, EMISAR. The EMISAR has been developed at the Section for Electromagnetic Systems (EMI), Ørsted•DTU Department, of the Technical University of Denmark, and it is an L- and C-band fully polarimetric and interferometric SAR [11]. The SAR system is installed on a Danish Air Force Gulfstream aircraft G-3. The SAR system is normally operated from an altitude of approximately $12.500 \mathrm{~m}$, the one-look spatial resolution is $2 \mathrm{~m}$ by $2 \mathrm{~m}$, and the ground range swath is approximately $12 \mathrm{~km}$. The processed data from this system are fully calibrated by using an internal calibration system. The image applied in this work is an Lband 13-look covariance matrix image at $5 \times 5 \mathrm{~m}$ ground pixel spacing, having a spatial resolution of approximately 8 x $8 \mathrm{~m}$. Fig. 2 shows an image of a Danish agricultural test area containing a number of agricultural fields, farms, hedges and forest areas.

In Figs. 3 and 4 are shown the results of applying the edge detector with the filter configuration $\mathrm{K}=\{9,1,3, \pi / 4\}$ to the image in Fig. 2. Only the diagonal elements are used in Fig. 3, i.e. only the backscatter coefficients. The result in Fig. 4 is for the azimuthally symmetric case, where also the hh,vv off-diagonal elements are included. 
The advantage of including the polarimetric information in the edge detection is clearly seen when comparing the results in Figs. 3 and 4 . Especially, the difference is seen for the edges between the fields $\mathrm{A}$ and $\mathrm{B}$, and $\mathrm{C}$ and $\mathrm{D}$, respectively, as indicated in Fig. 2. In these cases, no edge is detected when only the backscatter coefficients are used in the edge detection (cf. Fig. 3), whereas the edge is detected when the polarimetric information is included (cf. Fig. 4). Fields A and B are winter wheat and spring barley, respectively. The winter wheat field has a relatively high phase difference between $\mathrm{HH}$ and $\mathrm{VV}$, probably because of double bounce scattering between the soil surface and the vegetation. The spring barley field has a low phase difference. Fields $\mathrm{C}$ and $\mathrm{D}$ are oats and peas fields, respectively. The oats field has a high phase difference and the peas field has a low phase difference.

\section{CONCLUSIONS}

A new constant false alarm rate (CFAR) edge detector for polarimetric SAR images has been developed using a newly developed test statistic for the complex Wishart distribution. Using polarimetric SAR data from the Danish polarimetric SAR, EMISAR, the advantage of including the polarimetric information in the edge detection is clearly shown.

\section{REFERENCES}

[1] Touzi, R., A. Lopès, and P. Bousquet, 1988, "A statistical and geometrical edge detector for SAR images", IEEE Trans. Geosci. Remote Sens., vol. 26, pp. 764-773.

[2] Madsen, S. N., 1986, Speckle theory: Modelling, analysis, and applications related to synthetic aperture radar data, $\mathrm{Ph} . \mathrm{D}$. thesis, Electromagnetics Institute, Technical University of Denmark.

[3] van Zyl, J. J., and F. T. Ulaby, 1990, "Scattering matrix representation for simple targets", in F. T. Ulaby and C. Elachi, Radar Polarimetry for Geoscience Applications, Artech House.

[4] Schou, J., W. Dierking, and H. Skriver, 2000, "Tensor based structure estimation in multi-channel images", IGARSS00, pp. $663-665$

[5] Caves, R. G., I. McConnell, R. Cook, and S. Quegan, 1996, "Multi-channel SAR segmentation: Algorithms and Applications", IEE Colloquium on Image Processing for Remote Sensing, no. 5, pp. 2/1-2/6.

[6] Conradsen, K., A. A. Nielsen, J. Schou, and H. Skriver, "A test statistic in the complex Wishart distribution and its application to change detection in polarimetric SAR data", unpublished, 2001

[7] Conradsen, K., A. A. Nielsen, J. Schou, and H. Skriver, "Change detection in polarimetric SAR data and the complex Wishart distribution", in these proceedings, 2001.

[8] Schou, J., H. Skriver, A. A. Nielsen, and K. Conradsen, "CFAR edge detector for polarimetric SAR images", unpublished, 2001

[9] Goodman, N. R., 1963, "Statistical analysis based on a certain multivariate complex Gaussian distribution (An introduction)", Ann. Math. Stat., vol. 34, pp. 152-177.

[10] Borgeaud, M. R., T. Shin, and J. A. Kong, 1987, "Theoretical models for polarimetric radar clutter", J. Electromagn. Waves Appl., vol. 1, pp. 73-89.
[11] Christensen, E. L., N. Skou, J. Dall, K. Woelders, A. Netterstrøm, J.H. Jørgensen, J. Granholm, and S.N. Madsen, 1998, "EMISAR: Polarimetric L- and C-band SAR", IEEE Trans. Geosci. Remote Sens., vol. 36, pp. 1852-1865

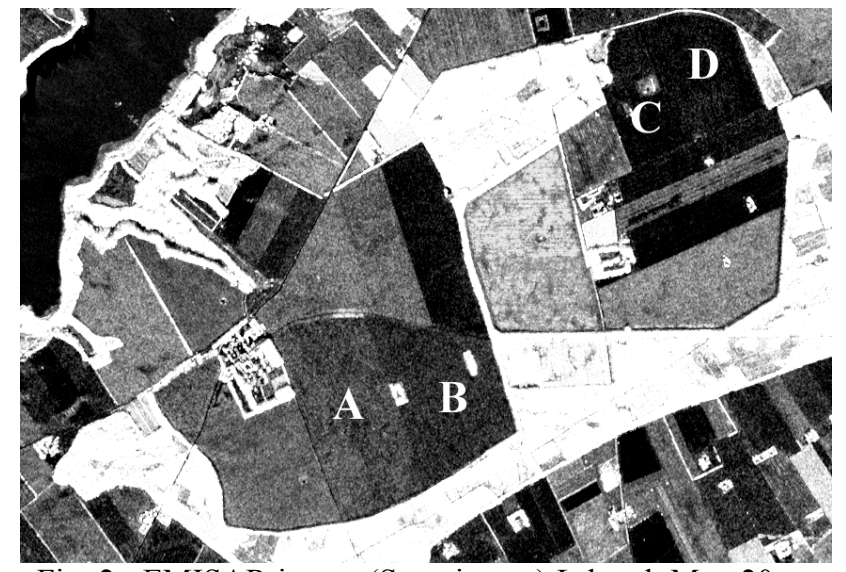

Fig. 2. EMISAR image (Span image) L-band, May 20, 1998. A: winter wheat, B: spring barley, C: oats, D: peas

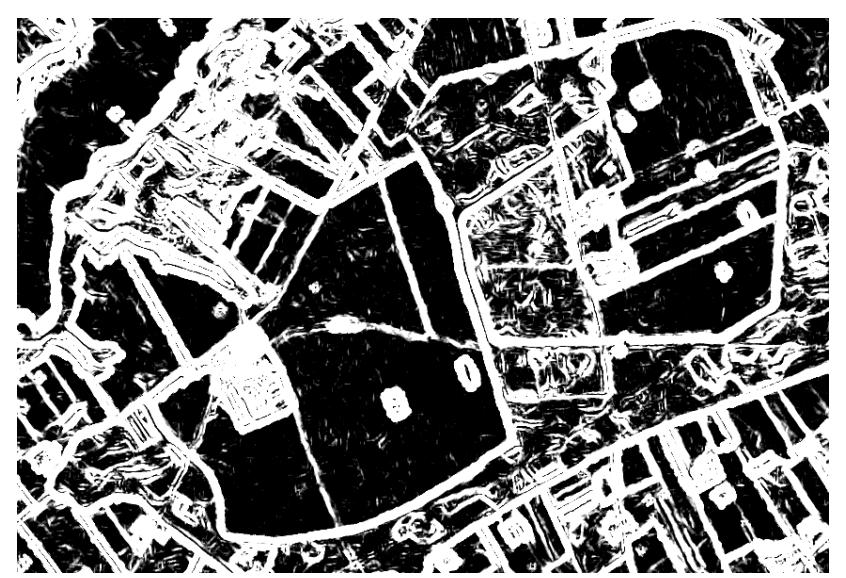

Fig. 3. Edge detection using diagonal elements

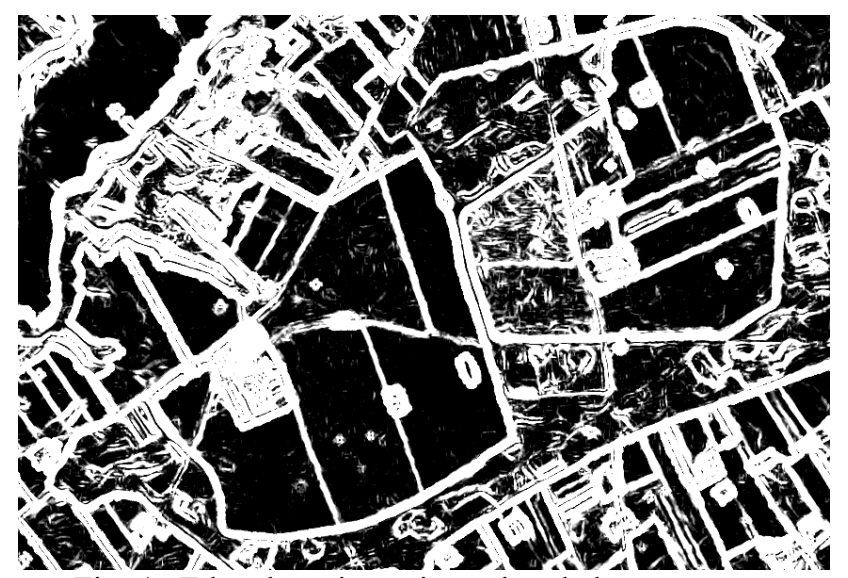

Fig. 4. Edge detection using azimuthal symmetry 